International Journal of Pure and Applied Mathematics

Volume 83 No. 4 2013, 613-621

ISSN: 1311-8080 (printed version); ISSN: 1314-3395 (on-line version)

url: http://www.ijpam.eu

doi: http://dx.doi.org/10.12732/ijpam.v83i4.10

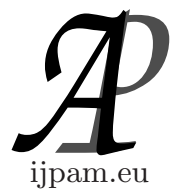

\title{
AMPLY WEAK SEMISIMPLE-SUPPLEMENTED MODULES
}

\author{
Figen Takil Mutlu \\ Department of Mathematics \\ Anadolu University \\ Eskisehir, 26470, TURKEY
}

\begin{abstract}
Let $R$ be a ring and $M$ be a right $R$-module. In this paper we will study various properties of amply weak semisimple-supplemented module. It is shown that: (1) every projective weakly semisimple-supplemented module is amply weak semisimple-supplemented; (2) if $M$ is an amply weak semisimplesupplemented module and satisfies DCC on weak semisimple-supplement submodules and on small submodules, then $M$ is Artinian; (3) an amply weak semisimple-supplemented module behaves well with respect to supplements and to homomorphic images.
\end{abstract}

AMS Subject Classification: 16D10, 16D60, 16D70, 16D99

Key Words: supplement submodule, weak semisimple-supplement submodule, amply weak semisimple-supplemented module

\section{Introduction}

Throughout this article, all rings are associative with unity and $R$ denotes such a ring. All modules are unital right $R$-modules unless indicated otherwise. Let $M$ be an $R$-module. $N \leq M$ will mean $N$ is a submodule of $M . \operatorname{Soc}(M)$, $\operatorname{End}(M)$ and $\operatorname{Rad}(M)$ will denote the Socle of $M$, the ring of endomorphisms of $\mathrm{M}$ and the Jacobson radical of $M$, respectively. The notions which are not explained here will be found in [7].

Received: January 7, 2013

(c) 2013 Academic Publications, Ltd. url: www.acadpubl.eu 
Recall that a module $M$ is called semisimple if it is a direct sum of simple submodules. A submodule $K$ is called small in $M$ (notation $K<<M$ ) if for every submodule $L$ in $M$, the equality $K+L=M$ implies $L=M$. A module $M$ is called hollow if every proper submodule of $H$ is small (see, [7]). Let $N$ and $L$ be submodules of $M . N$ is called a supplement of $L$ in $M$ if $N$ is a minimal element in the set of submodules $K \subset M$ with $M=K+L$ (see,[3]). In ([4], Definition 4.4, p.56) $M$ is called supplemented if any submodule of $M$ has a supplement in $M$.

In early years, supplemented modules and the other generalization, amply supplemented modules appeared in Helmut Zöschinger's works ([9], [10], [11], [12]). After Zöschinger, many authors (see for example [2], [5], [6] and [8]) studied on variations of supplemented modules. This paper is based on another variation of supplemented modules. We say that a submodule $N$ of $M$ has ample weak semisimple-supplements in $M$ if, for every $L \subseteq M$ with $N+L=$ $M$, there exists a weak semisimple-supplement $S$ of $N$ with $S \subseteq L$. We say that $M$ is amply weak semisimple-supplemented module if every submodule of $M$ has ample weak semisimple-supplements in $M$. We proved that every projective weak semisimple-supplemented module is amply weak semisimplesupplemented. It is shown that if $M$ is an amply weak semisimple-supplemented module and satisfies DCC on semisimple-supplement submodules and on small submodules, then $M$ is Artinian. Moreover, it is proven that an amply weak semisimple-supplemented module behaves well with respect to supplements and to homomorphic images.

In this section, we discuss the concept of semisimple-supplement submodules and we give some properties of such type submodules.

Definition 1. Let $M$ be an $R$-module, $N$ and $S$ be two submodules of M. $S$ is called semisimple-supplement of $N$ in $M$ if $N+S=M, N \cap S<<S$ and $\operatorname{Soc}(S)=S$.

Since $S$ is semisimple, every submodule of $S$ is a direct summand. If $S \cap$ $N<<S$, then $S \cap N=0$. Hence, $S$ being a semisimple-supplement of $N$, we have $M=N \oplus S, S$ is semisimple and $S$ is the minimal element in the set of submodules $K \subset M$ with $M=K+N$.

Definition 2. Let $M$ be an $R$-module. We say that $M$ is semisimplesupplemented if all submodules of $M$ has a semisimple-supplement in $M$.

Definition 3. Let $M$ be an $R$-module and $N \subseteq M$. If, for every $L \subseteq M$ with $N+L=M$, there exists a semisimple-supplement $S$ of $N$ with $S \subseteq L$, then we say that $\mathrm{N}$ has ample semisimple-supplements in $M$.

Definition 4. Let $M$ be an $R$-module. If every submodule of $M$ has 
ample semisimple-supplements in $M$, then $M$ is called amply semisimple-supplemented module.

It is clear that every amply semisimple-supplemented module is amply supplemented.

Proposition 5. Let $M$ be an $R$-module. Then the following statements are equivalent.

(a) $M$ is semisimple.

(b) $M$ is semisimple-supplemented.

(c) $M$ is amply semisimple-supplemented.

Proof. (a) $\Longrightarrow(\mathrm{b})$. It is clear.

(b) $\Longrightarrow(\mathrm{c})$. Let $M=N+L$. Since $M$ is semisimple-supplemented, there exists a semisimple supplement $S$ of $N$ in $M$. Then $M=N \oplus S$. Hence $M=(N+L) \cap(N \oplus S)=N \oplus(L \cap S)$. By the minimality of $S, L \cap S=S$, and hence $S \subseteq L$. Thus $N$ has ample semisimple supplement $S$ with $S \subseteq L$.

(c) $\Longrightarrow$ (a). Let $N \leq M$. Since $M$ is amply semisimple-supplemented module, there exists a semisimple supplement $S$ of $N$ in $M$. Then $S+N=M$ and $S \cap N<<S$. Since $S$ is semisimple, every submodule of $S$ is a direct summand. So $S \cap N=0$ and hence $S \oplus N=M$. Thus $M$ is semisimple.

Definition 6. Let $M$ be an $R$-module, $N S$ be two submodules of $M . S$ is called weak semisimple-supplement of $N$ in $M$ if $N+S=M, N \cap S<<M$ and $\operatorname{Soc}(S)=S$.

Definition 7. Let $M$ be an $R$-module. We say that a submodule $S \subset M$ is a weak semisimple-supplement if it is a weak semisimple-supplement for some submodule $N \subset M$.

Definition 8. Let $M$ be an $R$-module. If every nonzero submodule of $M$ has a weak semisimple-supplements in $M$, then $M$ is called a weakly semisimplesupplemented module or briefly a WSS-module.

It is clear that every semisimple-supplemented module is weakly semisimple supplemented.

Proposition 9. Let $M$ be an $R$-module, $N$ be a submodule of $M$ where $S$ be a weak semisimple-supplement of $N$ in $M$. Then the following statements are hold.

(1) If $K+S=M$ for some $K \subset N$, then $S$ is also a weak semisimplesupplement of $K$ in $M$. 
(2) If $M$ is finitely generated, then $S$ is also finitely generated.

(3) If $K<<M$, then $S$ is a weak semisimple-supplement of $N+K$ in $M$.

(4) For $K \subset N,(S+K) / K$ is a weak semisimple-supplement of $N / K$ in $M / K$.

Proof. (1) By the definition of weak semisimple-supplement, $N+S=M$, $N \cap S<<M$ and $S$ is semisimple. If $K+S=M$ for some $K \subset N$, then $K \cap S \subseteq N \cap S<<M$. Therefore $S$ is a weak semisimple-supplement of $K$ in $M$.

(2) From $([7], 41.1(2))$.

(3) Let $X \leq S$ and $N+K+X=M$. Since $K<<M, N+X=M$ and $N \cap X \subseteq N \cap S<<M$. By the minimality of $S, X=S$. Then $S$ is a weak semisimple-supplement of $N+K$ in $M$.

(4) By the definition of weak semisimple-supplement, $M=S+N, S \cap N<<$ $M$ and $S$ is semisimple. Hence $M=S+N+K$. Therefore $M / K=$ $N / K+[(S+K) / K]$. Now, we show that $(N / K) \cap[(S+K) / K]<<M / K$. Let $[(N / K) \cap[(S+K) / K]]+T / K=M / K$ and $K \subset T$. Then $[N \cap(S+$ $K)]+T=M$ and by modular law $K+(N \cap S)+T=M$. Since $N \cap S<<M$ and $K \subset T, T=T+K=M$. Hence $(N / K) \cap[(S+K) / K]<<M / K$. Thus $(S+K) / K$ is a supplement of $N / K$. Finally, since $S$ is semisimple, $(S+K) / K$ is semisimple submodule of $M / K$.

Lemma 10. Let $M$ be an $R$-module and $M_{1}, M_{2}, \ldots, M_{n}$ be submodules of $M$. Then $M_{1} \oplus M_{2} \oplus \cdots \oplus M_{n}$ is WSS-module if and only if every $M_{i}$ $(1 \leq i \leq n)$ is WSS-module.

Proof. Let $M=M_{1} \oplus M_{2} \oplus \cdots \oplus M_{n}$. To prove WSS-module it is sufficient by induction on $n$ to prove this when $n=2$. Thus suppose $n=2$.

Assume that $M$ is WSS-module. Let $N_{1} \oplus N_{2} \leq M=M_{1} \oplus M_{2}$. By assumption $N_{1}$ has a weak semisimple-supplement $S_{1}$ in $M_{1}$ and $N_{2}$ has a weak semisimple-supplement $S_{2}$ in $M_{2}$. Then $N_{1}+S_{1}=M_{1}, N_{1} \cap S_{1}<<M_{1}$, $N_{2}+S_{2}=M_{2}$ and $N_{2} \cap S_{2}<<M_{2}$. Hence

$$
M=M_{1} \oplus M_{2}=\left(N_{1}+S_{1}\right) \oplus\left(N_{2}+S_{2}\right)=\left(N_{1} \oplus N_{2}\right)+\left(S_{1} \oplus S_{2}\right),
$$

and

$$
\left(N_{1} \oplus N_{2}\right) \cap\left(S_{1} \oplus S_{2}\right) \subseteq\left(S_{1} \cap\left(N_{1} \oplus M_{2}\right)\right)+\left(S_{2} \cap\left(N_{1} \oplus M_{2}\right)\right)<<M_{1} \oplus M_{2}
$$


Since $S_{1}$ and $S_{2}$ are semisimple, $S_{1} \oplus S_{2}$ is semisimple. Hence $S_{1} \oplus S_{2}$ is weak semisimple-supplement of $N_{1} \oplus N_{2}$. Thus $M=M_{1} \oplus M_{2}$ is WSS-module.

Conversely, assume that $M_{1}$ and $M_{2}$ are WSS-module. Let $L \leq M_{1}$. By assumption $L \oplus M_{2}$ has a weak semisimple-supplement $S$ in $M$. Then $(L \oplus$ $\left.M_{2}\right)+S=M$ and $\left(L \oplus M_{2}\right) \cap S<<M$. Hence

$$
M_{1}=M_{1} \cap\left(\left(L \oplus M_{2}\right)+S\right)=L+\left(M_{1} \cap S\right),
$$

and

$$
L \cap S=L \cap\left(M_{1} \cap S\right) \subseteq\left(L \oplus M_{2}\right) \cap S<<M .
$$

Hence $L \cap\left(M_{1} \cap S\right)<<M_{1}$. Note that $M_{1} \cap S$ is semisimple since it is a submodule of semisimple submodule $S$. Thus $M_{1} \cap S$ is a weak semisimplesupplement of $L$ in $M_{1}$.

\section{Amply Weak Semisimple-Supplemented Modules}

In this section, we discuss the concept of amply weak semisimple-supplemented modules and we give some properties of such type modules.

Definition 11. Let $M$ be an $R$-module and $N \subseteq M$. If, for every $L \subseteq M$ with $N+L=M$, there exists a weak semisimple-supplement $S$ of $N$ with $S \subseteq L$, then we say that $\mathrm{N}$ has ample weak semisimple-supplements in $M$.

Definition 12. Let $M$ be an $R$-module. If every submodule of $M$ has ample weak semisimple-supplements in $M$, then $M$ is called an amply weak semisimple-supplemented module or briefly an AWSS-module.

Proposition 13. Every AWSS-module is WSS-module.

Proof. Let $M$ be an AWSS-module and $N$ be a submodule of $M$. Then $N+M=M$. Since $M$ is AWSS-module, $M$ contains a weak semisimplesupplement of $N$. Hence $M$ is WSS-module.

Proposition 14. Let $M$ be an $R$-module. If every submodule of $M$ is a WSS-module, then $M$ is AWSS-module.

Proof. Let $L, N \leq M$ and $M=N+L$. By assumption, there is a weak semisimple-supplement submodule $S$ of $L \cap N$ in $L$. Then $(L \cap N)+S=L$ and $(L \cap N) \cap S=N \cap S<<L$. Thus $N \cap S<<M$ and $S+N \geq S+(L \cap N)=L$ and hence $S+N \geq N+L=M$. Therefore $M=S+N$, as required. 
Proposition 15. Every factor module of an AWSS-module is AWSSmodule.

Proof. Let $M$ be an AWSS-module and $M / K$ be any factor module of $M$. Let $N / K \subseteq M / K$. For $L / K \subseteq M / K$, let $N / K+L / K=M / K$. Then $N+L=$ $M$. Since $M$ is AWSS-module, there exists a weak semisimple-supplement $S$ of $N$ with $S \subseteq L$. By Proposition $9(4),(S+K) / K$ is a weak semisimplesupplement of $N / K$ in $M / K$. Since $(S+K) / K \subseteq L / K, N / K$ has ample weak semisimple-supplements in $M / K$. Thus $M / K$ is AWSS-module.

Corollary 16. Every homomorphic image of an AWSS-module is AWSSmodule.

Proof. Let $M$ be an AWSS-module. Since every homomorphic image of $M$ is isomorphic to a factor module of $M$, every homomorphic image of $M$ is AWSS-module by Proposition 15 .

Proposition 17. Every supplement submodule of an AWSS-module is AWSS-module.

Proof. Let $M$ be an AWSS-module and $S$ be any supplement submodule of $M$. Then there exists a submodule $N$ of $M$ such that $S$ is a supplement of $N$. Let $L \subseteq S$ and $L+S^{\prime}=S$ for $S^{\prime} \subseteq S$. Then $N+L+S^{\prime}=M$. Since $M$ is AWSS-module, $N+L$ has a weak semisimple-supplement $S^{\prime \prime}$ in $M$ with $S^{\prime \prime} \subseteq S^{\prime}$. In this case $(N+L)+S^{\prime \prime}=M$. Since $L+S^{\prime \prime} \subseteq S$ and $S$ is a supplement of $N$ in $M, L+S^{\prime \prime}=S$. On the other hand, since $L \cap S^{\prime \prime} \subseteq(N+L) \cap S^{\prime \prime}<<M$, $L \cap S^{\prime \prime}<<M$. Hence $L$ has ample weak semisimple-supplements in $S$. Thus $S$ is AWSS-module.

Corollary 18. Every direct summand of an AWSS-module is AWSSmodule.

Proof. Let $M$ be an AWSS-module. Since every direct summand of $M$ is supplement in $M$, then by Proposition 17, every direct summand of $M$ is AWSS-module.

A module $M$ is said to be $\pi$-projective if, for every two submodules $N, L$ of $M$ with $L+N=M$, there exists $f \in \operatorname{End}(M)$ with $\operatorname{Im} f \leq L$ and $\operatorname{Im}(1-f) \leq$ $N$, see [7].

Theorem 19. Let $M$ be a WSS-module and $\pi$-projective module. Then $M$ is AWSS-module. 
Proof. Let $N \leq M$ and $L+N=M$ for $L \leq M$. Since $M$ is WSS-module, there exists a weak semisimple-supplement $S$ of $N$ in $M$. Then $N+S=M$, $N \cap S<<M$ and $S$ is semisimple. Since $M$ is $\pi$-projective, there exists an endomorphism $f$ such that $f(M) \leq L$ and $(1-f)(M) \leq N$. Note that $f(N) \subseteq N$ and $(1-f)(L) \subseteq L$. Then

$$
M=f(M)+(1-f)(M) \leq f(N \oplus S)+N=N+f(S) .
$$

Let $n \in N \cap f(S)$. Then there exists $s \in S$ with $n=f(s)$. In this case $s-n=s-$ $f(s)=(1-f)(s) \in N$ and then $s \in N$. Hence $s \in N \cap S$ and $N \cap f(S) \subseteq f(N \cap S)$. Since $N \cap S<<M$, then by Lemma ([7], 19.3(4)) $f(N \cap S)<<f(M)$. Then $N \cap f(S) \leq f(N \cap S)<<M$. Since $S$ is semisimple, $f(S)$ is semisimple. Hence $f(S)$ is a weak semisimple-supplement of $N$ in $M$. Since $f(S) \leq L, N$ has ample weak semisimple-supplements in $M$. Thus $M$ is AWSS-module.

Corollary 20. Every projective and WSS-module is AWSS-module.

Proof. Since every projective module is $\pi$-projective, every projective and WSS-module is AWSS-module by theorem 19.

Corollary 21. Let $M_{1}, M_{2}, \cdots, M_{n}$ be projective modules. Then $\underset{i=1}{\oplus} M_{i}$ is AWSS-module if and only if for every $1 \leq i \leq n, M_{i}$ is AWSS-module.

Proof. $(\Longrightarrow)$ It is clear from Corollary 18.

$(\Longleftarrow)$ Since, for every $1 \leq i \leq n, M_{i}$ is AWSS-module, $M_{i}$ is WSS-module. Then $\underset{i=1}{\bigoplus} M_{i}$ is also WSS-module by Lemma 10. Since, for every $1 \leq i \leq n, M_{i}$ is projective, $\bigoplus_{i=1}^{n} M_{i}$ is also projective. Then $\underset{i=1}{\bigoplus_{1}} M_{i}$ is AWSS-module by Corollary 20.

Corollary 22. Let $R$ be a ring. Then the following statements are equivalent.

(a) $R$ is weakly semisimple-supplemented.

(b) $R$ is amply weak semisimple-supplemented.

(c) Every finitely generated R-module is AWSS-module.

Proof. $(a) \Longleftrightarrow(b)$. Clear from Corollary 20.

$(a) \Longleftrightarrow(c)$. Clear from Corollary 16 and Corollary 21 . 
Theorem 23. ([1],Theorem 5) Let $R$ be any ring and $M$ be a module. Then $\operatorname{Rad}(M)$ is Artinian if and only if $M$ satisfies DCC on small submodules.

Proposition 24. Let $M$ be an R-module. If $M$ is an AWSS-module and satisfies DCC on weak semisimple-supplement submodules and on small submodules then $M$ is Artinian.

Proof. Let $M$ be an AWSS-module which satisfies DCC on weak semisimplesupplement submodules and on small submodules. Then $\operatorname{Rad}(M)$ is Artinian by Theorem 23. It suffices to show that $M / \operatorname{Rad}(M)$ is Artinian. Let $N$ be any submodule of $M$ containing $\operatorname{Rad}(M)$. Then there exists a weak semisimplesupplement $S$ of $N$ in $M$. Hence $M=N+S, N \cap S<<M$. Since $N \cap S \leq$ $\operatorname{Rad}(M), M / \operatorname{Rad}(M)=(N / \operatorname{Rad}(M)) \oplus((S+\operatorname{Rad}(M)) / \operatorname{Rad}(M))$ and so every submodule of $M / \operatorname{Rad}(M)$ is a direct summand. Therefore $M / \operatorname{Rad}(M)$ is semisimple.

Now suppose that $\operatorname{Rad}(M) \leq N_{1} \leq N_{2} \leq N_{3} \leq \cdots$ is an ascending chain of submodules of $M$. Because $M$ is AWSS-module, there exists a descending chain of submodules $S_{1} \geq S_{2} \geq S_{3} \geq \cdots$ such that $S_{i}$ is a weak semisimplesupplement of $N_{i}$ in $M$ for each $i \geq 1$. By hypothesis, there exists a positive integer $t$ such that $S_{t}=S_{t+1}=S_{t+2}=\cdots$. Because $M / \operatorname{Rad}(M)=N_{i} / \operatorname{Rad}(M) \oplus$ $\left(S_{i}+\operatorname{Rad}(M)\right) / \operatorname{Rad}(M)$ for all $i \geq t$, it follows that $N_{t}=N_{t+1}=\cdots$. Thus $M / \operatorname{Rad}(M)$ is Noetherian, and hence finitely generated. So $M / \operatorname{Rad}(M)$ is Artinian, as desired.

Corollary 25. Let $M$ be a finitely generated AWSS-module. If $M$ satisfies $D C C$ on small submodules, then $M$ is Artinian.

Proof. Since $M / \operatorname{Rad}(M)$ is semisimple and $M$ is finitely generated, $M / \operatorname{Rad}(M)$ is Artinian. Now that $M$ satisfies DCC on small submodules, $\operatorname{Rad}(M)$ is Artinian by Theorem 23. Thus $M$ is Artinian.

\section{References}

[1] I. Al-Khazzi, P.F. Smith, Modules with chain conditions on superfluous submodules, Comm. Algebra, 19 (1991), 2331-2351.

[2] G.F. Birkenmeier, F. Takıl Mutlu, C. Nebiyev, N. Sokmez, A. Tercan, Goldie*-supplemented Modules, Glasgow Math. J., 52A (2010), 41-52.

[3] J. Clark, C. Lomp, N. Vanaja, R. Wisbauer, Lifting Modules: Supplements and Projectivity in Module Theory, Birkhäuser Verlag, Basel, Switzerland (2006). 
[4] S.H. Mohamed, B.J. Muller, Continuous and Discrete Modules, London Mathematical Society Lecture Note Series, 147, Cambridge University Press, Cambridge (1990).

[5] Y. Talebi, A. Mahmoudi, On Rad- $\oplus$-supplemented modules, Thai Journal of Mathematics, 9, No. 2 (2011), 373-381.

[6] Y. Wang, N. Ding, Generalized supplemented modules, Taiwanese Journal of Mathematics, 10, No. 6 (2006), 1589-1601.

[7] R. Wisbauer, Foundations of Module and Ring Theory, Gordon and Breach, Philadelphia (1991).

[8] F. Yüzbaşı Eryılmaz, Ş. Eren, On (cofinitely) generalized amply weak supplemented modules, International Journal of Pure and Applied Mathematics, 76, No. 3 (2012), 333-342.

[9] H. Zöschinger, Komplementierte Moduln über Dedekindringen, Journal of Algebra, 29 (1974), 42-56.

[10] H. Zöschinger, Komplemente als direkte Summanden, Arch. Math. (Basel), 25 (1974), 241-253.

[11] H. Zöschinger, Komplemente als direkte Summanden II, Arch. Math. (Basel), 38, No. 4 (1982), 324-334.

[12] H. Zöschinger, Komplemente als direkte Summanden III, Arch. Math. (Basel), 46, No. 2 (1986), 125-135. 
